\section{Migração interna e a distribuição da mortalidade por doença de Chagas, Brasil, 1981/1998}

\author{
Internal migration and distribution of Chagas \\ disease mortality, Brazil, 1981-1998
}

\author{
1 Universidade Estadual \\ de Montes Claros, \\ Montes Claros, Brasil. \\ 2 Departamento de Medicina \\ Preventiva, Universidade \\ Federal de São Paulo, \\ São Paulo, Brasil. \\ Correspondência \\ L. F. Marcopito \\ Departamento de Medicina \\ Preventiva, Universidade \\ Federal de São Paulo. \\ Rua Borges Lagoa 1341, \\ São Paulo, SP \\ 04038-034, Brasil. \\ marcopito@medprev.epm.br
}

\begin{abstract}
In Brazil, mortality from Chagas disease occurs even in regions classified as free of vector transmission. Because death rates refer to residents, and considering that a huge migratory movement has occurred inside the country, this study was intended to quantify the contribution of Brazilian internal migration to overall mortality from Chagas disease, from 1981 to 1998. If the PAHO Southern Cone Initiative actually achieved its objectives, one could expect declining death rates and increasing age at death from this cause. The results showed that out of 68,936 deaths in Brazilians with known birthplace, 32,369 (32\%) occurred in people born in States other than those of their current residence (range: from $0.3 \%$ in Rio Grande do Sul to 100\% in Roraima and Amapá). Most (67\%) of the deaths in migrants occurred in individuals born in the States of Minas Gerais (51\%) and Bahia (16\%). Death rates in residents showed a consistent decline in the Southeast, South, and Central West of the country, but not in the Northeast and North, where median age at death was the lowest.
\end{abstract}

Chagas Disease; Internal Migration; Mortality
João Augusto Guimarães Drumond 1 Luiz Francisco Marcopito 2

\section{Introdução}

O Brasil já foi grande pólo de atração de imigrantes estrangeiros, que inicialmente se destinavam a substituir a mão-de-obra escrava na lavoura cafeeira no interior do Estado de São Paulo 1. Antes de 1920 os imigrantes estrangeiros predominavam, passando, desde então, a ser substituídos pelos chamados "trabalhadores nacionais" 2 em decorrência do movimento migratório interno brasileiro iniciado ao fim do século XIX, primeiro do Nordeste para a região de fronteira, especialmente a amazônica ${ }^{3}$. A partir de 1930 o governo federal passou a impor severas restrições à entrada de estrangeiros no país e a incentivar a migração interna nacional, particularmente a de brasileiros do Nordeste 4 . Após a II Guerra Mundial o movimento migratório interno brasileiro tornou-se incontrolável: estima-se que, de 1950 a 1996, mais de 40 milhões deixaram o campo em migrações inter e intra-regionais 5 .

Por sua vez, a doença de Chagas é reconhecidamente uma endemia rural. Embora a transmissão possa ocorrer, artificialmente, por transfusão sangüínea e, naturalmente, por via transplacentária ou digestiva, a transmissão vetorial sempre foi considerada a mais importante no Brasil 6 . A faixa territorial brasileira tradicionalmente considerada como de transmissão vetorial da doença inclui grandes áreas das regiões Centro-oeste, Sul, Sudeste e Nordeste, mas não 
a maior parte da Região Norte. No entanto, há mortalidade por doença de Chagas em residentes de praticamente todas as Unidades da Federação (UF) do país 7 .

Considerando que as taxas de mortalidade referem-se a residentes, que houve imenso movimento migratório interno no país, que a transmissão natural da doença de Chagas está sendo eliminada 8 , justifica-se um estudo que procure quantificar a participação dos migrantes brasileiros no obituário por doença de Chagas e determinar se houve mudanças nas taxas de mortalidade e idade dos óbitos.

\section{Método}

Estudo de mortalidade em série temporal de seis triênios, com dados secundários. Os arquivos eletrônicos de declarações de óbitos em residentes de cada uma das 27 UF, correspondentes aos anos de 1981 a 1998 (exceto o Estado de Tocantins - criado em 1988) estavam armazenados em CD-ROM fornecidos pelo extinto CENEPI (Centro Nacional de Epidemiologia). Havia ao todo 479 arquivos no formato "dbc", que foram descompactados em "dbf" e, em ambiente SPSS (SPSS Inc., Chicago, Estados Unidos) transformados de "dbf" para "sav", com a seleção de variáveis de interesse.

Foram estudadas quatro variáveis: causa básica de morte, naturalidade do falecido, idade em que ocorreu o óbito, e UF de residência do falecido. A causa básica de morte selecionada foi "doença de Chagas", codificada pela Classificação Estatística Internacional de Doenças e Problemas Relacionados à Saúde (CID), nona revisão (CID-9) e décima revisão (CID-10). Pela CID-9, códigos 086.0 a 086.2 e 086.9; pela CID10, códigos B57. 0 a B57. 5.

Calcularam-se taxas de mortalidade padronizadas por idade (usando como referência a estrutura etária brasileira do triênio 1981/1983), do período total 1981/1998 e por triênios. As medianas de idade dos óbitos também foram calculadas, bem como a mortalidade proporcional por doença de Chagas em nativos e migrantes.

\section{Resultados}

A Figura 1 mostra as taxas médias anuais de mortalidade por doença de Chagas em residentes das UF, ajustadas por idade, no total do período 1981/1998. Observa-se que os residentes em Goiás, Distrito Federal e Minas Gerais, nessa ordem, foram os que mais risco apresenta- ram de morrer por doença de Chagas no total do período de observação. É necessário ressaltar que as taxas apresentadas na Figura 1 referem-se a pessoas residentes nas UF, independente da naturalidade dos falecidos.

Dos 105.476 óbitos por doença de Chagas ocorridos no total do período, 4.171 (4\%) referiam-se a pessoas com naturalidade imprecisa ou estrangeiras. Excluídas estas, chega-se à Tabela 1, que apresenta os óbitos por doença de Chagas em brasileiros com naturalidade conhecida. Observa-se que a porcentagem total de óbitos em brasileiros não naturais da UF de residência foi $32 \%$, variando de $0,3 \%$ (no Rio Grande do Sul) a 100\% (em Roraima e Amapá, em que pese o reduzido número de óbitos por doença de Chagas nessas duas UF e nas demais da região Norte). Chama atenção que na maioria das UF do Nordeste, em Minas Gerais e no Rio Grande do Sul predominam os óbitos por doença de Chagas em naturais das próprias UF, o oposto ocorrendo no Distrito Federal e no Paraná e, em menor escala, no Rio de Janeiro, Espírito Santo e Santa Catarina. Em Goiás e São Paulo, UF com altas taxas médias de mortalidade por doença de Chagas no período 1981/1998, respectivamente $37,8 \%$ e $51,1 \%$ dos óbitos em brasileiros ocorreram em naturais de outras UF.

A Tabela 1 também dá conta de 92,8\% de todos os óbitos ocorridos no país no período 1981/1998 (tirante os estrangeiros e aqueles com naturalidade imprecisa). Desses óbitos gerais em brasileiros com naturalidade conhecida, 19,6\% ocorreram em não naturais da UF de residência, cifra que variou de $5,7 \%$ (no Rio de Janeiro) a $34,7 \%$ (no Piauí). Observa-se que, na Região Norte, a porcentagem de óbitos por doença de Chagas em migrantes $(82,7 \%)$ foi muito maior do que a dos óbitos gerais em migrantes (12\%). Excetuado o Maranhão, nos Estados do Nordeste, em Minas Gerais $(96,2 \%)$ e no Rio Grande do Sul $(99,7 \%)$ a porcentagem de óbitos por doença de Chagas em nativos foi maior do que a dos óbitos gerais em não naturais da própria UF.

Quanto à mortalidade proporcional por doença de Chagas, observa-se na Tabela 1 que há diferenças marcantes em nativos e migrantes de algumas UF como Rondônia, Tocantins, São Paulo, Paraná e todas as unidades da Região Centro-oeste - das quais se destacam Goiás e Distrito Federal.

Por outro lado, observa-se na Tabela 2 que, dos 32.369 brasileiros com naturalidade conhecida e falecidos por doença de Chagas em outras UF que não a de nascimento, 16.521 (51\%) eram do Estado de Minas Gerais, 5.170 (16\%) da Bahia, 2.471 (7,6\%) de São Paulo, 1.786 (5,5\%) 
de Goiás e 1.498 (4,6\%) de Pernambuco. Dos restantes $4.923(15,2 \%)$ procedentes das demais UF, quase três quartos (3.655) também eram nordestinos.

Ainda na Tabela 2, observa-se que no Estado de São Paulo ocorreu o maior número de óbitos (13.579) por doença de Chagas em migrantes internos no período 1981/1998, o grande contingente $(94,3 \%)$ procedente de Minas $\mathrm{Ge}$ rais $(7.380$, ou $54,3 \%)$ e do Nordeste (5.425, ou $40 \%)$. Em Goiás (6.527) e no Distrito Federal (4.213) também houve elevado número de óbitos por essa causa em brasileiros não naturais dessas UF, a maioria também proveniente de Minas Gerais e do Nordeste (bem como de Goiás no Distrito Federal). No Paraná (4.194), predominaram os óbitos de mineiros e paulistas entre os migrantes brasileiros.

A Tabela 3 dá conta de 94.961 (93,7\%) dos 101.305 óbitos por doença de Chagas no total do período 1981/1998, e mostra as medianas de idade da morte na região ou UF com as maiores contribuições no obituário total, de acordo com a naturalidade e a residência dos falecidos. Chama atenção o estado de Goiás, onde tanto os nativos como os migrantes exibiram medianas $\geq 60$ anos de idade, mas entre os goianos que faleceram no Distrito Federal a mediana foi bem menor $(55,0)$. Também é digna de nota a observação de que, entre os falecidos no Estado do Paraná, os nativos dessa UF apresentaram mediana $(49,0)$ muito inferior às dos naturais do Nordeste $(57,0)$, de Minas Gerais $(56,0)$ e de São Paulo $(61,0)$. Para finalidade de comparação, a Tabela 3 exibe também as medianas de idade da morte por todas as causas.

A Tabela 4 refere-se a óbitos em residentes. Com relação às taxas de mortalidade, verificase que as regiões Sudeste, Sul e Centro-oeste mostraram declínio sustentado no período 1981/ 1998 (com redução relativa em torno de 50\%), porém situação oposta ocorreu na Região Norte durante os cinco primeiros triênios de observação, enquanto a Região Nordeste apresentou o padrão mais estável, com resistência a quedas. Com relação às medianas de idade dos óbitos por doença de Chagas, percebe-se aumento progressivo no período de observação (exceto na Região Norte no triênio 1996/ 1998).

Durante todo o período de observação foram registrados apenas 44 óbitos em menores de um ano de idade, nenhum destes residentes da Região Norte.
Figura 1

Taxas médias anuais (ajustadas por idade *) de mortalidade (por milhão) por doença de Chagas, por Unidade da Federação (UF), em residentes, no período 1981/1998.

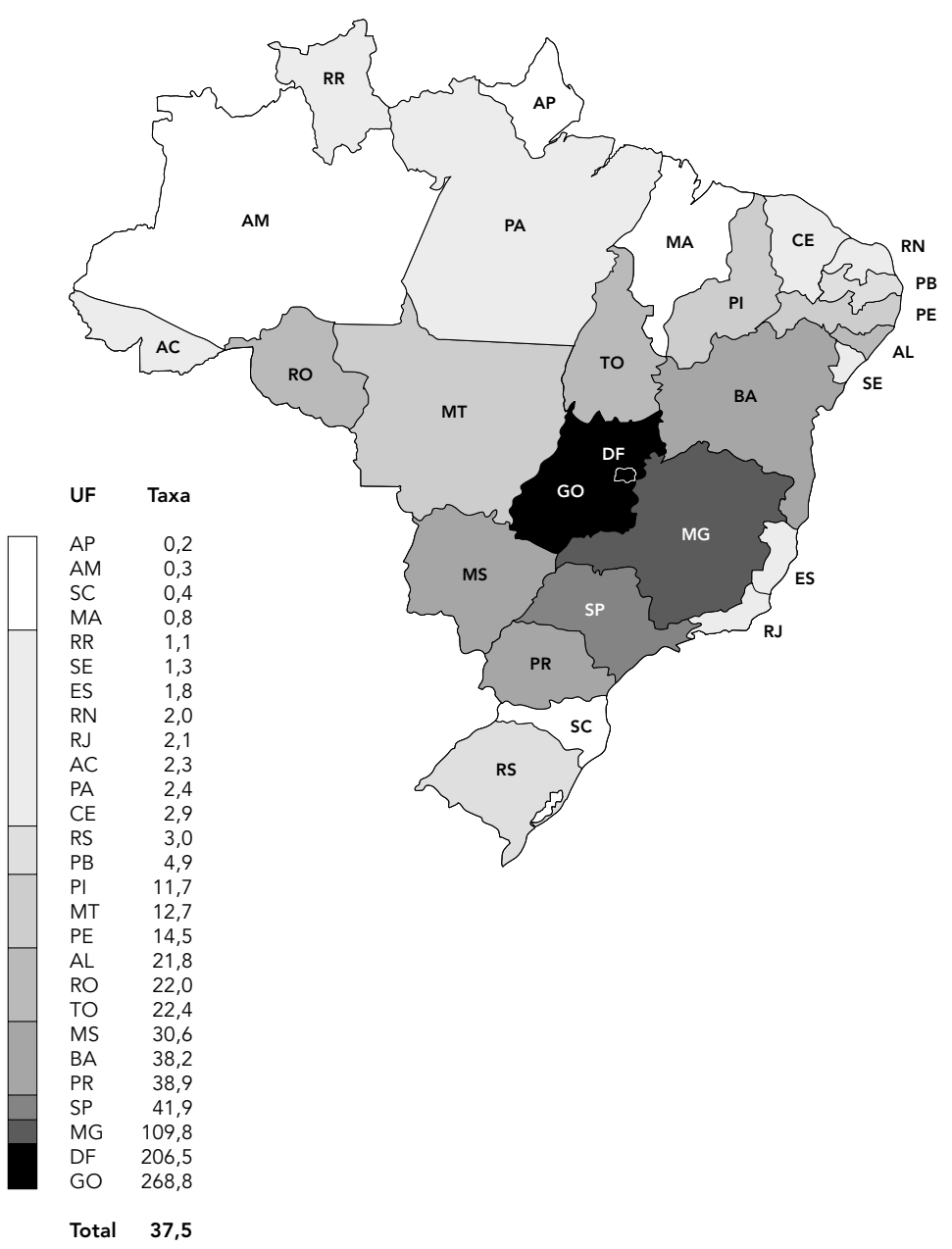

$\mathrm{AC}=$ Acre $\mathrm{AL}=$ Alagoas $; \mathrm{AM}=$ Amazonas $; \mathrm{AP}=$ Amapá $\mathrm{BA}=$ Bahia $; \mathrm{CE}=$ Ceará; $\mathrm{DF}=$ Distrito Federal; $\mathrm{ES}=$ Espírito Santo; $\mathrm{GO}=$ Goiás; $\mathrm{MA}=$ Maranhão; MG = Minas Gerais; MS = Mato Grosso do Sul; MT = Mato Grosso; PA = Pará; $\mathrm{PB}=$ Paraíba; $\mathrm{PE}=$ Pernambuco; PI = Piauí; PR = Paraná; RJ = Rio de Janeiro; $\mathrm{RN}=$ Rio Grande do Norte; RO = Rondônia; RR = Roraima; RS = Rio Grande do Sul; $\mathrm{SC}=$ Santa Catarina; $\mathrm{SE}=$ Sergipe $; \mathrm{SP}=$ São Paulo; $\mathrm{TO}=$ Tocantins.

* Ajuste pela estrutura etária brasileira do triênio 1981/1983;

** Os óbitos em residentes do Tocantins foram contados em Goiás até 1989

A taxa média de Tocantins, portanto, refere-se ao período de nove anos (1990/1998).

\section{Discussão}

Este estudo estava inicialmente planejado para cobrir o período 1979/2002, no entanto ocorreu o seguinte: primeiro, as tabulações de 1979 e 1980 , respectivamente, mostraram que $44,7 \%$ e $28,7 \%$ de todos os falecidos no Ceará estavam registrados como naturais do Amazonas, fato que não encontra respaldo na história da mi- 
Tabela 1

Número e porcentagem de óbitos por doença de Chagas, por todas as causas, e mortalidade proporcional por doença de Chagas em brasileiros residentes das Unidades da Federação (UF) e com naturalidade conhecida, de acordo com a naturalidade. Brasil, $1981 / 1998$.

\begin{tabular}{|c|c|c|c|c|c|c|c|c|c|c|c|}
\hline \multirow{3}{*}{$\begin{array}{l}\text { UF de residência } \\
\text { do falecido (e total) } \\
\text { por região) }\end{array}$} & \multicolumn{4}{|c|}{ Óbitos por doença de Chagas } & \multicolumn{4}{|c|}{ Óbitos por todas as causas } & \multicolumn{3}{|c|}{ Mortalidade proporcional (\%) } \\
\hline & \multicolumn{2}{|c|}{$\begin{array}{l}\text { Naturais da } \\
\text { própria UF }\end{array}$} & \multicolumn{2}{|c|}{$\begin{array}{l}\text { Naturais de } \\
\text { outras UFs }\end{array}$} & \multicolumn{2}{|c|}{$\begin{array}{l}\text { Naturais da } \\
\text { própria UF }\end{array}$} & \multicolumn{2}{|c|}{$\begin{array}{l}\text { Naturais de } \\
\text { outras UFs }\end{array}$} & \multirow[t]{2}{*}{$\begin{array}{l}\text { Naturais da } \\
\text { própria UF }\end{array}$} & \multirow[t]{2}{*}{$\begin{array}{l}\text { Naturais de } \\
\text { outras UFs }\end{array}$} & \multirow[t]{2}{*}{ Total } \\
\hline & Número & $\%$ & Número & $\%$ & Número & $\%$ & Número & $\%$ & & & \\
\hline Região Norte & 116 & 17,3 & 556 & 82,7 & 458.830 & 88,0 & 62.480 & 12,0 & * & 0,9 & 0,1 \\
\hline Rondônia & 3 & 1,2 & 245 & 98,8 & 26.560 & 92,8 & 2.055 & 7,2 & * & 11,9 & 0,9 \\
\hline Acre & 4 & 30,8 & 9 & 69,2 & 26.180 & 81,3 & 6.034 & 18,7 & * & 0,1 & * \\
\hline Amazonas & 5 & 50,0 & 5 & 50,0 & 116.824 & 87,7 & 16.391 & 12,3 & * & * & * \\
\hline Roraima & ** & $\star \star$ & 2 & 100,0 & 8.369 & 91,0 & 830 & 9,0 & ** & 0,2 & * \\
\hline Pará & 31 & 18,3 & 138 & 81,7 & 258.020 & 88,8 & 32.624 & 11,2 & * & 0,4 & 0,1 \\
\hline Amapá & ** & ** & 1 & 100,0 & 12.141 & 91,3 & 1.156 & 8,7 & ** & 0,1 & * \\
\hline Tocantins ${ }^{\star \star \star}$ & 73 & 31,9 & 156 & 68,1 & 10.736 & 76,0 & 3.390 & 24,0 & 0,7 & 4,6 & 1,6 \\
\hline Região Nordeste & 10.890 & 95,6 & 505 & 4,4 & 3.253 .565 & 72,9 & 1.210 .824 & 27,1 & 0,3 & * & 0,3 \\
\hline Maranhão & 38 & 67,9 & 18 & 32,1 & 181.731 & 75,3 & 59.749 & 24,7 & * & * & * \\
\hline Piauí & 468 & 95,1 & 24 & 4,9 & 119.254 & 65,3 & 63.487 & 34,7 & 0,4 & * & 0,3 \\
\hline Ceará & 305 & 93,3 & 22 & 6,7 & 412.338 & 73,9 & 145.782 & 26,1 & 0,1 & * & 0,1 \\
\hline Rio Grande do Norte & 76 & 85,4 & 13 & 14,6 & 165.193 & 75,3 & 54.282 & 24,7 & * & * & * \\
\hline Paraíba & 284 & 92,8 & 22 & 7,2 & 336.022 & 70,8 & 138.535 & 29,2 & 0,1 & * & 0,1 \\
\hline Pernambuco & 1.651 & 90,6 & 171 & 9,4 & 831.032 & 77,9 & 235.379 & 22,1 & 0,2 & 0,1 & 0,2 \\
\hline Alagoas & 801 & 90,9 & 80 & 9,1 & 259.649 & 70,2 & 109.998 & 29,8 & 0,3 & 0,1 & 0,2 \\
\hline Sergipe & 27 & 93,1 & 2 & 6,9 & 119.959 & 68,3 & 55.658 & 31,7 & * & * & * \\
\hline Bahia & 7.240 & 97,9 & 153 & 2,1 & 828.387 & 70,4 & 347.954 & 29,6 & 0,9 & * & 0,6 \\
\hline Região Sudeste & 44.909 & 74,5 & 15.410 & 25,5 & 5.296 .204 & 82,8 & 1.100 .428 & 17,2 & 0,8 & 1,4 & 0,9 \\
\hline Minas Gerais & 31.805 & 96,2 & 1.260 & 3,8 & 1.538 .440 & 67,1 & 753.664 & 32,9 & 2,1 & 0,2 & 1,4 \\
\hline Espírito Santo & 25 & 30,5 & 57 & 69,5 & 194.900 & 70,7 & 80.658 & 29,3 & * & 0,1 & * \\
\hline Rio de Janeiro & 75 & 12,7 & 514 & 87,3 & 1.197 .002 & 94,3 & 71.714 & 5,7 & * & 0,7 & * \\
\hline São Paulo & 13.004 & 48,9 & 13.579 & 51,1 & 2.365 .862 & 92,4 & 194.392 & 7,6 & 0,5 & 7,0 & 1,0 \\
\hline Região Sul & 2.035 & 32,6 & 4.213 & 67,4 & 1.776 .455 & 86,4 & 279.471 & 13,6 & 0,1 & 1,5 & 0,3 \\
\hline Paraná & 1.398 & 25,0 & 4.194 & 75,0 & 450.704 & 83,3 & 90.314 & 16,7 & 0,3 & 4,6 & 1,0 \\
\hline Santa Catarina & 10 & 37,0 & 17 & 63,0 & 292.814 & 81,2 & 67.913 & 18,8 & * & * & * \\
\hline Rio Grande do Sul & 627 & 99,7 & 2 & 0,3 & 1.032 .937 & 89,5 & 121.244 & 10,5 & 0,1 & * & 0,1 \\
\hline Região Centro-oeste & 10.986 & 48,5 & 11.685 & 51,5 & 384.649 & 83,7 & 74.728 & 16,3 & 2,9 & 15,6 & 4,9 \\
\hline Mato Grosso do Sul & 115 & 14,5 & 676 & 85,5 & 77.935 & 87,6 & 11.001 & 12,4 & 0,1 & 6,1 & 0,9 \\
\hline Mato Grosso & 51 & 15,9 & 269 & 84,1 & 60.298 & 79,1 & 15.927 & 20,9 & 0,1 & 1,7 & 0,4 \\
\hline Goiás & 10.736 & 62,2 & 6.527 & 37,8 & 220.193 & 85,3 & 38.011 & 14,7 & 4,9 & 17,2 & 6,7 \\
\hline Distrito Federal & 84 & 2,0 & 4.213 & 98,0 & 26.223 & 72,8 & 9.789 & 27,2 & 0,3 & 43,0 & 11,9 \\
\hline Brasil & 68.936 & 68,0 & 32.369 & 32,0 & 11.169 .703 & 80,4 & 2.727 .931 & 19,6 & 0,6 & 1,2 & 0,7 \\
\hline
\end{tabular}

$\star<0,1 \%$;

** Denota zero;

*** Os óbitos em residentes do Tocantins foram contados em Goiás até 1989

As porcentagens de Tocantins, portanto, referem-se ao período de nove anos (1990/1998). 
Número de óbitos por doença de Chagas entre migrantes brasileiros, de acordo com a Unidade da Federação (UF) de naturalidade e de residência. Brasil, 1981/1998.

\begin{tabular}{|c|c|c|c|c|c|c|c|c|c|c|c|}
\hline \multirow{3}{*}{$\begin{array}{l}\text { Região e UF } \\
\text { de naturalidade } \\
\text { dos falecidos }\end{array}$} & \multicolumn{11}{|c|}{ Região e UF de residência dos falecidos } \\
\hline & \multirow[t]{2}{*}{ Norte } & \multirow[t]{2}{*}{ Nordeste } & \multicolumn{3}{|c|}{ Sudeste } & \multicolumn{2}{|c|}{ Sul } & \multicolumn{3}{|c|}{ Centro-Oeste } & \multirow[t]{2}{*}{ Total } \\
\hline & & & $\begin{array}{l}\text { Minas } \\
\text { Gerais }\end{array}$ & São Paulo & Outras UF & Paraná & Outras UF & Goiás & $\begin{array}{l}\text { Distrito } \\
\text { Federal }\end{array}$ & Outras UF & \\
\hline \multicolumn{12}{|l|}{ Norte } \\
\hline Todas & 6 & 5 & 6 & 23 & 2 & 4 & * & 70 & 21 & 5 & 142 \\
\hline \multicolumn{12}{|l|}{ Nordeste } \\
\hline Piauí & 29 & 15 & 10 & 231 & 8 & 10 & * & 122 & 289 & 7 & 721 \\
\hline Ceará & 13 & 34 & 21 & 246 & 26 & 41 & $\star$ & 91 & 101 & 18 & 591 \\
\hline Paraíba & 2 & 134 & 35 & 247 & 43 & 24 & 1 & 70 & 137 & 8 & 701 \\
\hline Pernambuco & 13 & 131 & 49 & 893 & 55 & 118 & * & 105 & 108 & 26 & 1.498 \\
\hline Alagoas & 3 & 48 & 15 & 628 & 26 & 141 & * & 19 & 13 & 41 & 934 \\
\hline Bahia & 47 & 20 & 269 & 2.995 & 181 & 309 & 1 & 801 & 446 & 101 & 5.170 \\
\hline Total & 163 & 445 & 480 & 5.425 & 348 & 671 & 2 & 1.385 & 1.196 & 208 & 10.323 \\
\hline \multicolumn{12}{|l|}{ Sudeste } \\
\hline Minas Gerais & 212 & 27 & $\star \star$ & 7.380 & 194 & 2.013 & 4 & 4.627 & 1.693 & 371 & 16.521 \\
\hline São Paulo & 46 & 14 & 366 & ** & 9 & 1.391 & 3 & 332 & 48 & 262 & 2.471 \\
\hline Outras UF & 8 & 3 & 69 & 88 & 7 & 45 & 1 & 24 & 33 & 6 & 284 \\
\hline Total & 266 & 44 & 435 & 7.468 & 210 & 3.449 & 8 & 4.983 & 1.774 & 639 & 19.276 \\
\hline \multicolumn{12}{|l|}{ Sul } \\
\hline Paraná & 22 & 2 & 12 & 480 & 1 & $\star \star$ & 4 & 7 & 2 & 20 & 550 \\
\hline Outras UF & 2 & * & 8 & 26 & 1 & 57 & 4 & 11 & 3 & 10 & 122 \\
\hline Total & 24 & 2 & 20 & 506 & 2 & 57 & 8 & 18 & 5 & 30 & 672 \\
\hline \multicolumn{12}{|l|}{ Centro-oeste } \\
\hline Total & 97 & 9 & 319 & 157 & 10 & 13 & 1 & 71 & 1.217 & 62 & 1.956 \\
\hline \multicolumn{12}{|l|}{ Brasil } \\
\hline Todas & 556 & 505 & 1.260 & 13.579 & 572 & 4.194 & 19 & 6.527 & 4.213 & 1.071 & 32.369 \\
\hline
\end{tabular}

* Denota zero;

** Não se aplica.

gração interna brasileira 5 . Isso pode ter ocorrido por erro de digitação, já que os códigos daquelas duas UF são muito parecidos (823 e 813). Segundo, a qualidade de registro do campo "naturalidade" sofreu grande deterioração a partir de 1999, tanto nos óbitos por todas as causas como nos óbitos por doença de Chagas. Assim, o presente trabalho restringiu-se ao período 1981/1998, quando a porcentagem de naturalidade indeterminada entre os falecidos por doença de Chagas no país ficou em 3,7\%, variando de cifras tão baixas quanto $0,0 \%$ (Acre e Amapá) a 18,2\% (Rondônia). Nas UF com maior contribuição porcentual no total de óbitos por doença de Chagas (Minas Gerais, São Paulo, Goiás, Bahia, Paraná e Distrito Federal, soma de $92,3 \%$ ), essa porcentagem ficou em $3,5 \%$, variando de 1,1\% em São Paulo a 9,3\% no Paraná.

A idade dos falecidos por doença de Chagas não se constituiu em obstáculo à realização deste trabalho, visto que apenas em $0,7 \%$ das declarações de óbito essa informação não estava disponível.

$\mathrm{Na}$ interpretação dos resultados deve-se levar em conta que este estudo de âmbito nacional tem sua validade prejudicada por alguns problemas: (a) a cobertura (razão entre óbitos informados e estimados) do Sistema de Infor- 
Medianas de idade da morte por doença de Chagas, números de óbitos* e medianas de idade da morte por todas as causas em região e Unidades da Federação (UF) selecionadas, por naturalidade e residência dos falecidos. Brasil, 1981/1998.

\begin{tabular}{|c|c|c|c|c|c|}
\hline \multirow[t]{2}{*}{ Região ou UF de residência } & \multicolumn{5}{|c|}{ Região ou UF de naturalidade } \\
\hline & Nordeste & Minas Gerais & São Paulo & Paraná & Goiás \\
\hline \multicolumn{6}{|l|}{ Nordeste } \\
\hline Mediana de idade da morte por doença de Chagas & 53,0 & $\star \star$ & ** & ** & ** \\
\hline Óbitos & 11.276 & ** & ** & 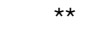 & ** \\
\hline Mediana de idade da morte por todas as causas & 56,0 & ** & ** & ** & ** \\
\hline \multicolumn{6}{|l|}{ Minas Gerais } \\
\hline Mediana de idade da morte por doença de Chagas & ** & 59,0 & ** & ** & ** \\
\hline Óbitos & ** & 31.669 & ** & ** & ** \\
\hline Mediana de idade da morte por todas as causas & ** & 59,0 & $\star \star$ & $\star \star$ & $\star \star$ \\
\hline \multicolumn{6}{|l|}{ São Paulo } \\
\hline Mediana de idade da morte por doença de Chagas & 51,0 & 55,0 & 59,0 & 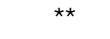 & ** \\
\hline Óbitos & 5.425 & 7.377 & 13.000 & ** & ** \\
\hline Mediana de idade da morte por todas as causas & 57,0 & 63,0 & 56,0 & ** & ** \\
\hline \multicolumn{6}{|l|}{ Paraná } \\
\hline Mediana de idade da morte por doença de Chagas & 57,0 & 56,0 & 61,0 & 49,0 & ** \\
\hline Óbitos & 670 & 2.011 & 1.391 & 1.396 & ** \\
\hline Mediana de idade da morte por todas as causas & 66,0 & 67,0 & 67,0 & 44,0 & 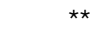 \\
\hline \multicolumn{6}{|l|}{ Goiás } \\
\hline Mediana de idade da morte por doença de Chagas & 63,0 & 62,0 & ** & ** & 60,0 \\
\hline Óbitos & 1.385 & 4.608 & ** & ** & 10.664 \\
\hline Mediana de idade da morte por todas as causas & 64,0 & 65,0 & $\star \star$ & $\star \star$ & 48,0 \\
\hline \multicolumn{6}{|l|}{ Distrito Federal } \\
\hline Mediana de idade da morte por doença de Chagas & 56,0 & 56,0 & ** & ** & 55,0 \\
\hline Óbitos & 1.195 & 1.693 & ** & ** & 1.201 \\
\hline Mediana de idade da morte por todas as causas & 57,0 & 58,0 & ** & ** & 50,0 \\
\hline
\end{tabular}

* Podem não coincidir com os números da Tabela 2, pela exclusão de alguns óbitos com idade desconhecida: ** Medianas não calculadas quando o número de óbitos por doença de Chagas nos 18 anos de observação foi inferior a 500 .

mações sobre Mortalidade varia com a região do país e, dentro desta, com a UF - por exemplo, em 1997/1998 a cobertura nacional foi estimada em $81 \%$, entretanto, nas UF com maior contribuição no obituário por doença de Chagas, as cifras situaram-se acima desse valor $(82,9 \%$ no Distrito Federal a $98 \%$ em São Paulo, exceto na Bahia, 60,6\%) 7; (b) a porcentagem de óbitos por causas mal definidas, que também é desigual entre as regiões, e se agrava fora das capitais das UF. No total do período 1979/1998, a porcentagem nacional situou-se em $18,7 \%$, e nas UF com maior contribuição no obituário por doença de Chagas as cifras situaram-se abaixo (Distrito Federal, 1,9\%; São Paulo, 6\%; Paraná, 13,4\%; Minas Gerais, 17,2\%) ou acima
(Goiás, 20,2\%; Bahia, 33,3\%) desse valor 7; (c) em estudos de mortalidade como este, realizados com dados secundários, um potencial problema é a causa básica da morte, cuja fidedignidade depende, entre os óbitos com assistência médica, da qualidade dessa assistência, do acesso a recursos diagnósticos e da importância que é conferida à declaração de óbito 9 .

Mesmo levadas em conta essas limitações, os resultados obtidos neste estudo são dignos de nota: Goiás, Distrito Federal e Minas Gerais foram as UF que apresentaram, nessa ordem, as maiores taxas médias anuais de mortalidade por doença de Chagas entre residentes no período. As taxas de mortalidade em residentes, todavia, contam apenas parte da história por- 
Taxas trienais de mortalidade (por milhão) por doença de Chagas, ajustadas por idade*, e medianas de idade da morte em residentes das grandes regiões. Brasil, 1981/1998.

\begin{tabular}{|c|c|c|c|c|c|c|c|c|c|c|c|c|}
\hline \multirow[t]{2}{*}{ Triênio } & \multicolumn{5}{|c|}{$\begin{array}{l}\text { Taxas trienais de mortalidade } \\
\text { Região de residência dos falecidos }\end{array}$} & \multicolumn{6}{|c|}{$\begin{array}{l}\text { Medianas de idade da morte } \\
\text { Região de residência dos falecidos }\end{array}$} & \multirow[t]{2}{*}{ Brasil } \\
\hline & Norte & Nordeste & Sudeste & Sul & Centro-oeste & & Norte & Nordeste & Sudeste & Sul & Centro-oeste & \\
\hline $1981 / 1983$ & 4,2 & 16,2 & 64,8 & 21,1 & 249,2 & 50,2 & 45,5 & 47,0 & 54,0 & 52,0 & 56,0 & 54,0 \\
\hline $1984 / 1986$ & 4,6 & 16,8 & 56,7 & 18,8 & 218,6 & 45,2 & 48,5 & 50,0 & 56,0 & 54,0 & 58,0 & 55,0 \\
\hline 1987/1989 & 4,8 & 16,7 & 51,3 & 15,9 & 180,6 & 40,6 & 52,0 & 51,0 & 57,0 & 57,0 & 60,0 & 57,0 \\
\hline 1990/1992 & 6,7 & 14,9 & 44,7 & 13,4 & 146,2 & 34,7 & 55,5 & 53,0 & 58,0 & 58,0 & 61,0 & 58,0 \\
\hline 1993/1995 & 7,1 & 15,7 & 41,0 & 13,1 & 136,5 & 32,8 & 62,0 & 55,0 & 60,0 & 60,0 & 61,0 & 60,0 \\
\hline 1996/1998 & 5,7 & 15,8 & 33,8 & 11,5 & 109,3 & 27,9 & 59,0 & 57,0 & 61,0 & 61,0 & 63,0 & 61,0 \\
\hline
\end{tabular}

* Ajuste pela estrutura etária brasileira do triênio 1981/1983.

que, de todos os óbitos por doença de Chagas em brasileiros com naturalidade conhecida $(96,1 \%$ do total de mortes por essa causa no período), $32 \%$ ocorreram em naturais de outras UF, cifra que variou de 0,3\% no Rio Grande do Sul a 100\% em Roraima e Amapá.

As UF com as maiores porcentagens de óbitos de naturais fora de suas fronteiras foram Minas Gerais (51\% daqueles 32\%), Bahia (16\%), São Paulo (7,6\%), Goiás $(5,5 \%)$ e Pernambuco $(4,6 \%)$. Entre esses óbitos fora das UF de naturalidade, a maioria das mortes em migrantes mineiros $(44,7 \%)$, baianos $(57,9 \%)$ e pernambucanos $(59,6 \%)$ ocorreu em São Paulo. Já a maioria dos óbitos em paulistas migrantes $(56,3 \%)$ foi no Paraná, e a maioria das mortes em goianos migrantes $(67,6 \%)$ aconteceu no Distrito Federal.

Por outro lado, na região Norte, $82,7 \%$ dos 556 óbitos por doença de Chagas em residentes brasileiros com naturalidade conhecida ocorreram em migrantes, cifra que se opõe diametralmente à da Região Nordeste, onde apenas $4,4 \%$ dos 11.395 óbitos nessas condições ocorreram em migrantes. Nessa pequena parcela, a maior contribuição percentual $(88,1 \%)$ veio de naturais de outras UF da própria região. Depreende-se daí que a migração originada em outras regiões do país foi importante (em porcentagens locais) para o Norte, mas insignificante para o Nordeste.

Em cifras nacionais, o movimento migratório de pessoas que vieram a falecer por doença de Chagas foi mais importante do Sudeste para o Sudeste, Centro-oeste e Sul, e do Nordeste para o Sudeste e o Centro-oeste. Em escala bem menor, do Centro-oeste para o Centro-oeste, do Sul para o Sudeste, e do Nordeste para o Nordeste e o Sul.
Esse movimento se reflete na mortalidade proporcional por doença de Chagas em migrantes de algumas UF, como as da Região Norte (sobretudo Rondônia e Tocantins), São Paulo, Paraná e as da Região Centro-oeste - todas acima da média nacional de $1,2 \%$.

As taxas de mortalidade por doença de Chagas em residentes, de 1981/1993 para 1996/1998, caíram muito nas regiões Sudeste, Sul e Centro-oeste, fenômeno que não ocorreu no Nordeste e Norte. Isso pode estar relacionado à conseqüência de esforços dirigidos mais à eliminação do Triatoma infestans, que é pouco freqüente ou inexistente nessas regiões 8 .

A taxa de mortalidade em residentes (forma habitual de descrição) não é um bom indicador para o controle da doença de Chagas em regiões reconhecidas como de transmissão natural por vetor, pois o numerador é muito afetado pelos óbitos em migrantes. O caso típico é o do Estado de São Paulo, onde apenas metade dos óbitos por doença de Chagas (anotados entre 1981/ 1998 em residentes) ocorreu em naturais da própria UF. Além disso, São Paulo foi a UF natal de 7,6\% de todos os óbitos por doença de Chagas registrados (no mesmo período) no território nacional.

Essa invasão/evasão de óbitos não pode simplesmente ser diminuída/somada (do/ao) numerador para a obtenção da taxa de mortalidade em nativos porque (a) não se conhece a população de nativos (denominador) e (b) não se sabe onde ocorreu a infecção: se na UF de naturalidade, se na UF de residência, ou se em trânsito durante a migração até a UF de residência onde ocorreu o óbito. Por isso, nem mesmo a mortalidade por doença de Chagas em estrangeiros não naturais das Américas poderia ser tomada como indício de transmissão autóctone na UF de residência do falecido. 
Há que se considerar, ainda, a chamada "migração de retorno" 10, que complicaria a interpretação da mortalidade por doença de Chagas em naturais da própria UF, já que a infecção poderia ter ocorrido durante o tempo em que o migrante permaneceu fora de sua UF de naturalidade - se a região de destino fosse de transmissão vetorial.

Com relação às medianas de idade de ocorrência dos óbitos por doença de Chagas em residentes, estas vêm aumentando em todo o país, mas as medianas da Região Nordeste teimam em ficar abaixo das medianas nacionais. Chama atenção o fato de que no Centro-oeste os óbitos por doença de Chagas se deram em idade mais avançada do que nas demais regiões durante todo o período 1981/1998, certamente influenciada pelos falecidos residentes em Goiás.

Os casos de Goiás e do Paraná merecem estudo especial: os goianos falecidos por doença de Chagas no Distrito Federal mostraram mediana de idade muito inferior à dos residentes, nativos e migrantes, naquela UF (êxodo dos casos mais graves para centro urbano com mais recursos de assistência médica?); os paranaenses exibiram mediana muito inferior à dos migrantes. Além disso, nesses dois estados as medianas de idade dos óbitos gerais em nativos foram muito inferiores às medianas dos óbitos por doença de Chagas.

A existência de óbitos por doença de Chagas em menores de um ano de idade, embora em pequeno número no período de estudo (44), sugere mais transmissão congênita do que autóctone na região de residência. Mesmo considerando que o diagnóstico da doença nessa idade seja de difícil realização, é digno de nota o fato de não ter havido qualquer óbito nessa faixa etária em residentes da Região Norte.

Segundo especialistas, as migrações transculturais são mais claramente o resultado de instabilidade social e da pressão da desigualdade entre regiões 11. O que move a jovem população rural para a migração são essencialmente preocupações profissionais 12 . A crise pode afetar o ritmo da migração, aumentando sua velocidade quando determinada população busca novos horizontes econômicos 13 .

Importantes transformações no perfil da saúde e da doença na população brasileira têm sido creditadas a migrações da área rural para a urbana, pois hábitos culturais e sociais vindos das áreas rurais são adotados pelos migrantes nas áreas urbanas, aumentando o risco de transmissão de doenças 14 . Em relação à doença de Chagas, havia a preocupação sobre a possibilidade de a migração aumentar o risco de urbanização da doença no Brasil 15, pela transmissão transfusional. Contudo, a implementação de efetiva política nacional de vigilância em bancos de sangue a partir dos anos 1980 alcançou níveis elevados de controle, atingindo 98\% em 1995 no país 16,17,18.

Nos últimos 15 anos, uma série de iniciativas multinacionais levou à redução significativa no impacto social e econômico da doença de Chagas nas Américas. No início dos anos 90, a doença de Chagas era classificada pelo Banco Mundial como a mais séria das doenças parasitárias na América Latina, com impacto sócioeconômico maior que o efeito das outras doenças parasitárias combinadas. O número estimado de pessoas infectadas era de 16-18 milhões, caindo para 11 milhões em 2002. As ações de controle passaram pelo combate ao vetor, controle dos bancos de sangue e, em muitas áreas, a observação da migração humana de áreas rurais endêmicas para as cidades onde a relação de transmissão via vetor é baixa 19 .

Atualmente, com as melhorias habitacionais realizadas nas áreas endêmicas e com a estratégia de monitoramento entomológico para identificar a presença do vetor e desencadear as ações de combate utilizando inseticidas específicos, essa doença encontra-se sob controle. Tal fato pode ser constatado com base no consolidado dos inquéritos sorológicos para a doença de Chagas realizados sistematicamente entre escolares (7-14 anos de idade) de todos os estados endêmicos, no período de 1989 a 1999. No consolidado de 244.770 amostras colhidas, apenas 329 foram positivas, resultando na prevalência média geral de $0,13 \% 8$.

Com esses resultados e a redução da área onde é encontrado o T. infestans, a Comissão Internacional de Especialistas constituída pela Organização Pan-Americana da Saúde (OPAS) e pelos países do Cone Sul, com a finalidade de avaliar a situação epidemiológica de cada país, conferiu o certificado de interrupção da transmissão vetorial pelo T. infestans a 11 Estados brasileiros: São Paulo (onde a transmissão havia sido interrompida na década de 1970) 20, Rio de Janeiro, Paraíba, Mato Grosso, Mato Grosso do Sul, Goiás, Minas Gerais, Pernambuco, Tocantins, Piauí 8 e, mais recentemente 21 , Rio Grande do Sul.

Autores 22 questionam, com razão, o significado da certificação conferida ao Brasil como "estando livre da doença de Chagas por Triatoma infestans", porque alguns Estados (Bahia e Paraná) possuem municípios ainda com infestação por esse vetor ${ }^{8}$. Ainda que a transmissão por T. infestans tivesse sido interrompida, como está em vias de ser 8 , isso pouco afetaria a 
transmissão vetorial na maior parte dos Estados do Nordeste (Maranhão, Ceará, Rio Grande do Norte, Alagoas e Sergipe) nem em alguns outros Estados (Espírito Santo e Santa Catarina), nos quais, apesar de pertencerem à área endêmica, não existe a espécie T. infestans 8 .

Já a Amazônia brasileira há muito tempo tem sido considerada área não endêmica para a doença de Chagas 23, mesmo sendo demonstrada a presença do Trypanosoma cruzi em diferentes animais silvestres 24 . Existem na região pelo menos 18 espécies de triatomíneos e, destas, 10 já foram identificados como infectadas pelo Tr. cruzi, mas ainda sem domiciliação nem hábitos antropofílicos 25 . Os maiores riscos para o estabelecimento de área endêmica nessa parte do país são a migração da população humana infectada para a área e a derrubada descontrolada da mata 23. Nessa região, por mais que tenha havido movimento migratório no tempo dos colonizadores portugueses (que ex- pulsaram os demais grupos europeus que tentavam se estabelecer na região amazônica) e também à época do Império (do ciclo das "drogas do sertão" para o ciclo da borracha), o estabelecimento na área foi relativamente pequeno se comparado ao que ocorreu a partir da década de 1960, com taxa de crescimento anual da população residente de 5\% em 1970/1980 e de $4,1 \%$ em 1980/1991, o dobro da taxa nacional 26. Assim, os efeitos sobre a mortalidade por doença de Chagas em residentes da região, se houver, devem ser esperados para o futuro.

A tripanossomíase americana é reconhecidamente uma endemia rural, portanto a mortalidade por essa doença em migrantes nacionais reflete em grande parte o êxodo dos campos por que passou o país nos últimos 60-100 anos. A questão da migração e doença de Chagas transfusional ou congênita, infelizmente, não pode ser avaliada por dados secundários de mortalidade, como os deste estudo.

\section{Resumo}

No Brasil, observa-se mortalidade por doença de Chagas até em áreas reconhecidas como livres da transmissão vetorial. Considerando que as taxas de mortalidade referem-se a residentes, e que houve imenso movimento migratório interno no país, este estudo objetiva quantificar a participação dos migrantes brasileiros no obituário por doença de Chagas de 1981 a 1998. Por outro lado, se os trabalhos da Iniciativa do Cone Sul alcançaram o sucesso que se propaga, espera-se que tenha havido redução das taxas de mortalidade e aumento na idade em que ocorrem os óbitos por essa causa. Dos 68.936 óbitos em brasileiros com naturalidade conhecida, 32.369 (32\%) foram em nascidos em outras Unidades da Federação que não a de residência do falecido, cifra que variou de 0,3\% no Rio Grande do Sul a 100\% em Roraima e Amapá. A maioria (67\%) desses óbitos em migrantes ocorreu em naturais de Minas Gerais (51\%) e Bahia (16\%). As taxas de mortalidade em residentes mostraram declínio sustentado no Sudeste, Sul e Centro-oeste, mas não no Nordeste e Norte, onde as idades medianas de morte foram as mais baixas.

Doença de Chagas; Migração Interna; Mortalidade

\section{Colaboradores}

J. A. G. Drumond fez o levantamento bibliográfico e redigiu o texto. L. F. Marcopito extraiu as informações dos bancos de dados, organizando-as em tabelas e figuras. 


\section{Referências}

1. Secretaria de Estado da Cultura de São Paulo. O Memorial do Imigrante. http://www.memorialdoimigrante.sp.gov.br (acessado em Abr/2001).

2. Camargo JF. O desenvolvimento da população paulista depois de 1886: a população alienígena no Estado de São Paulo. In: Camargo JF, organizador. Crescimento da população no Estado de São Paulo e seus aspectos econômicos. São Paulo: IPE/USP; 1981. p. 113-53.

3. Cavalcanti H, Guillen I. Atravessando fronteiras: movimentos migratórios na história do Brasil. http://www.imaginario.com.br (acessado em Mar/ 2003).

4. Paiva OC. Breve história da hospedaria de imigrantes e da imigração para São Paulo. São Paulo: Secretaria de Estado da Cultura de São Paulo; 2000. (Série Resumos, 7).

5. Camarano AA, Abramovay R. Êxodo rural, envelhecimento e masculinização no Brasil: panorama dos últimos cinqüenta anos. Revista Brasileira de Estudos Populacionais 1998; 15:45-65.

6. Secretaria Nacional de Vigilância em Saúde, Ministério da Saúde. Doença de Chagas aguda: manual prático de subsídio à notificação obrigatória no SINAN. http://portal.saude.gov.br (acessado em Ago/2005).

7. Departamento de Informação e Informática do Sistema Único de Saúde. Estatísticas de mortalidade. http://tabnet.datasus.gov.br/cgi/sim (acessado em Abr/2005).

8. Departamento de Análise de Situação de Saúde, Secretaria de Vigilância em Saúde, Ministério da Saúde. Saúde Brasil 2004: uma análise da situação de saúde. Brasília: Ministério da Saúde; 2004.

9. Mameri CP. Tendências populacionais: óbitos por causas mal definidas. Conjuntura Demográfica 1990; 10:7-10.

10. Ribeiro JT, Carvalho JA. Perspectivas das migrações internas no Brasil: a imigração para Minas Gerais no período 1981-1991, com especial enfoque na migração de retorno. In: XI Encontro Nacional de Estudos Populacionais da ABEP. http: //www.abep.nepo.unicamp.br (acessado em Set/ 2004).

11. Hull D. Migration, adaptation, and illness: a review. Soc Sci Med 1979; 13A:25-36.

12. Detang-Dessendre C. Reciprocal link between exit from unemployment and geographical mobility. Environ Plan A 1999; 31:1417-31.

13. Martine G, Neiva IC, Macedo M. Migração, crise e outras agruras. http://www.abep.nepo.unicamp. br (acessado em Set/2004).

14. Mott KE, Desjeux P, Moncayo A, Ranque P, de Raadt P. Parasitic diseases and urban development. Bull World Health Organ 1990; 68:691-8.
15. Baruffa G. A doença de Chagas no Rio Grande do Sul, Brasil. Vittalle 1986; 2:9-18.

16. World Health Organization. Chagas disease: interruption of transmission. Wkly Epidemiol Rec 1997; 72:1-5.

17. World Health Organization. Doença de Chagas. In: United Nations Development Program/World Bank/World Health Organization, editor. TDR Thirteenth Programme Report. Geneva: World Health Organization; 1997. p. 112-23.

18. Souza HM, Ramirez LE, Bordin JO. Doença de Chagas transfusional: medidas de controle. In: Dias JCP, Coura JR, organizadores. Clínica e terapêutica da doença de Chagas: uma abordagem prática para o clínico geral. Rio de Janeiro: Editora Fiocruz; 1997. p. 429-43.

19. Dias JCP, Silveira AC, Schofield CJ. The impact of Chagas disease control in Latin America: a review. Mem Inst Oswaldo Cruz 2002; 97:603-12.

20. Superintendência de Controle de Endemias. Programa de controle da doença de Chagas no Estado de São Paulo. http://www.sucen.sp.gov.br (acessado em Dez/2005).

21. Pan American Health Organization. XIVth meeting of the Intergovernmental Commission of the Southern Cone (INCOSUR) for the elimination of T. infestans and the interruption of transfusional American trypanosomiasis. Santa Cruz de la Sierra: Pan American Health Organization; 2005.

22. Ramos Jr. AN, Carvalho DM. Os diferentes significados da certificação conferida ao Brasil como estando livre da doença de Chagas. Cad Saúde Pública 2001; 17:1403-12.

23. Valente SAS, Valente VC, Neto HF. Considerations on the epidemiology and transmission of Chagas disease in the Brazilian Amazon. Mem Inst Oswaldo Cruz 1999; 94 Suppl 1:395-8.

24. Barretto MP. Reservatórios do Trypanosoma cruzi nas Américas. Rev Bras Malariol Doenças Trop 1964; 16:527-52.

25. Coura JR, Junqueira ACV, Boia MN, Fernandes O. Chagas disease: from bush to huts and houses. Is it the case of the Brazilian Amazon? Mem Inst Oswaldo Cruz 1999; 94 Suppl 1:379-84.

26. Brasil MC. A migração interestadual na região Norte: a década de 70. In: Anais do X Encontro da Associação Brasileira de Estudos Populacionais. v. 2. Belo Horizonte: Associação Brasileira de Estudos Populacionais; 1996. p. 639-58.

Recebido em 23/Set/2005

Versão final reapresentada em 06/Jan/2006 Aprovado em 17/Jan/2006 